\title{
A Distribution-Independent Bound on the Level of Confidence in the Result of a Measurement
}

\section{W. Tyler Estler}

National Institute of Standards and Technology,

Gaithersburg, MD 20899-0001
The Bienaymé-Chebyshev Inequality provides a quantitative bound on the level of confidence of a measurement with known combined standard uncertainty and assumed coverage factor. The result is independent of the detailed nature of the probability distribution that characterizes knowledge of the measurand.
Key words: level of confidence; measurement; probability; uncertainty.

Accepted: September 10, 1997

\section{Introduction}

The ISO Guide to the Expression of Uncertainty in Measurement [1] and the NIST adaption [2] recommend that the result of a measurement of a quantity $Y=f\left(x_{1}, x_{2}, \cdots, x_{N}\right)$ be reported as $Y=y \pm U$, where $y$ is the estimate (or expectation) of $Y$ and $U$ is an expanded uncertainty defined by $U=k u_{\mathrm{c}}(y)$. Here $u_{\mathrm{c}}(y)$ is the combined standard uncertainty and $k$ is a coverage factor chosen to produce an interval having a level of confidence close to a desired value. For uncorrelated input quantities, the combined standard uncertainty is the positive square root of the variance

$$
u_{\mathrm{c}}^{2}(y)=\sum_{i=1}^{N} u_{i}^{2}(y),
$$

where the terms $u_{i}^{2}(y) \equiv\left(\partial f / \partial x_{i}\right)^{2} u^{2}\left(x_{i}\right)$ are weighted variances associated with the probability distributions that characterize one's knowledge of the input quantities $x_{i}$.
The level of confidence associated with a particular choice of coverage factor $k$ is interpreted to mean that the true value of $Y$ may be expected to lie in the interval $y \pm k u_{\mathrm{c}}(y)$ with an integrated, or cumulative, probability $P(k)$. The Guide gives an extended discussion of the problem of establishing the relation between $k$ and $P$, the details of which depend on the exact (and generally unknown) probability distribution associated with one's knowledge of the measurand $Y$.

This note describes a very general and useful bounding relation, long known to professional statisticians, that is free of these details. An interesting collection of such relations was compiled and described by I. R. Savage [3]. Our motivation derives from the growing acceptance and use of the Guide in engineering metrology and industrial quality control, and a perceived need for a wider exposure to some of the fundamental ideas of probability theory. 


\section{The Bienaymé-Chebyshev Inequality}

This simple but general result was first derived by the French mathematician I. J. Bienaymé (1853) and rediscovered by P. L. Chebyshev (1867). We follow the development of Kenney and Keeping [4]. Consider a general probability density function $p(Y)$ that satisfies the basic requirements of being normalized and having finite mean $y$ and variance $\sigma^{2}$ :

$$
\begin{gathered}
1=\int_{-\infty}^{+\infty} p(Y) \mathrm{d} Y \\
y=\int_{-\infty}^{+\infty} Y p(Y) \mathrm{d} Y \\
\sigma^{2}=\int_{-\infty}^{+\infty}(Y-y)^{2} p(Y) \mathrm{d} Y .
\end{gathered}
$$

Now let $a$ be an arbitrary positive constant. The probability that $|Y-y| \geq a$ is given by:

$$
\operatorname{Pr}(|Y-y| \geq a)=\int_{R} p(Y) \mathrm{d} Y,
$$

where $R$ denotes the set of values of $Y$ that satisfy the stated inequality. Now clearly

$$
\sigma^{2} \geq \int_{R}(Y-y)^{2} p(Y) \mathrm{d} Y \geq a^{2} \int_{R} p(Y) \mathrm{d} Y,
$$

so that from Eqs. (3) and (4) we have immediately:

$$
\operatorname{Pr}(|Y-y| \geq a) \leq \frac{\sigma^{2}}{a^{2}} .
$$

This result is known as the Bienaymé-Chebyshev Inequality. Setting $a=k \sigma$, Eq. (5) becomes:

$$
\operatorname{Pr}(|Y-y| \geq k \sigma) \leq \frac{1}{k^{2}} .
$$

We thus see that independent of the detailed nature of the distribution $p(Y)$, the probability that the true value of $Y$ will differ from its expected (or measured) value $y$ by as much as $k$ standard deviations is not more than $1 / k^{2}$. Now for $k \leq 1$, this is not very informative, but for larger values of $k$ it becomes quite interesting. It tells us, for example, that not less than $8 / 9 \approx 89 \%$ of the probability associated with a measurement of $Y$ is contained in the interval $y \pm 3 \sigma$, whatever the distribution $p(Y)$.
For a probability distribution known to be normal (or Gaussian), the corresponding result would be $99.7 \%$. While this is a significant improvement in the level of confidence, the lower value may be completely adequate for the measurement task at hand, while avoiding the need for nit-picking over the details of the exact form of the distribution $p(Y)$.

\section{Acknowledgments}

We thank Drs. Keith Eberhardt and Steven D. Phillips for valuable comments and suggestions. This work was supported in part by the U.S. Air Force CCG Program and the NIST Competence Program in Computational Metrology.

\section{References}

[1] International Organization for Standardization (ISO), Guide to the Expression of Uncertainty in Measurement, ISO, Geneva (1995).

[2] Barry N. Taylor and Chris E. Kuyatt, Guidelines for Evaluating and Expressing the Uncertainty of NIST Measurement Results, NIST Technical Note 1297 (1994).

[3] I. R. Savage, Probability Inequalities of the Tchebyscheff Type, J. Res. Natl. Bur. Stand. (U.S.) 65B, 211-222 (1961).

[4] J. F. Kenney and E. S. Keeping, Mathematics of Statistics, Part Two, 2nd ed., Van Nostrand Co., New York (1951) pp. 84-85.

About the author: W. Tyler Estler is a physicist in the Precision Engineering Division of the Manufacturing Engineering Laboratory at NIST. The National Institute of Standards and Technology is an agency of the Technology Administration, U.S. Department of Commerce. 